\title{
Highly Efficient Small Interfering RNA Delivery to Primary Mammalian Neurons Induces MicroRNA-Like Effects before mRNA Degradation
}

\author{
Thomas J. Davidson, ${ }^{1}$ Sivan Harel, ${ }^{1}$ Valerie A. Arboleda, ${ }^{1}$ Giselle F. Prunell, ${ }^{1}$ Michael L. Shelanski, ${ }^{1,3}$ Lloyd A. Greene, ${ }^{1,3}$ \\ and Carol M. Troy ${ }^{1,2,3}$ \\ Departments of ${ }^{1}$ Pathology and ${ }^{2}$ Neurology, Taub Institute for the Study of Alzheimer's Disease and the Aging Brain, and ${ }^{3}$ Center for Neurobiology and \\ Behavior, Columbia University College of Physicians and Surgeons, New York, New York, 10032
}

\begin{abstract}
The study of protein function in neurons has been hindered by the lack of highly efficient, nontoxic methods of inducing RNA interference in such cells. Here we show that application of synthetic small interfering RNA (siRNA) linked to the vector peptide Penetratin 1 results in rapid, highly efficient uptake of siRNA by entire populations of cultured primary mammalian hippocampal and sympathetic neurons. This treatment leads to specific knock-down of targeted proteins within hours without the toxicity associated with transfection. In contrast to current methods, our technique permits study of protein function across entire populations with minimal disturbance of complex cellular networks. Using this technique, we found that protein knock-down (evident after $6 \mathrm{hr}$ ) precedes any decrease in targeted message (evident after $24 \mathrm{hr}$ ), suggesting an early, translational repression by perfectly targeted siRNAs.
\end{abstract}

Key words: primary neurons; hippocampal neurons; siRNA; Penetratin1; transduction peptides; RNA interference

\section{Introduction}

Genetic manipulations of cultured cells have provided deep insight into the biology of normal and diseased states by allowing the molecular dissection of important biological pathways. Primary neuronal cultures, although particularly attractive to researchers for their similarities to living systems, present special challenges. Transgenic animals are a valuable source of genetically altered cells for primary cultures, but creation of knock-outs is time- and resource-intensive, and knocked-out proteins can be compensated for during development (Troy et al., 2001). Recently, RNA interference (RNAi), a sequence-specific silencing of mRNA by stretches of homologous double-stranded RNA, has been shown to function across a wide range of organisms, including mammals (Hannon, 2002). In addition to its power as a tool for biologists, RNAi is thought to play an important role in the regulation of gene expression. MicroRNAs (miRNAs), endogenously expressed short double-stranded RNAs, have been shown to be developmentally regulated and to share much of the same machinery as small interfering RNA (siRNA)-induced gene silencing (Hutvagner and Zamore, 2002). In the nervous system,

Received July 19, 2004; accepted Sept. 21, 2004.

This work was supported by grants from the National Institutes of Health (C.M.T., L.A.G.,M.L.S.) and the Muscular Dystrophy Association (C.M.T.). We thank Greg Hannon for helpful discussions and particularly for suggesting the use of blunt double-stranded RNA. We thank Gene Marcantonio, Chris Petersen, and Bevil Conway for critical reading of this manuscript and Marissa Maclin for excellent technical assistance.

Correspondence should be addressed to Dr. Carol M. Troy, Columbia University College of Physicians and Surgeons, 630 West 168th Street, New York, NY 10032. E-mail: cmt2@columbia.edu.

T. J. Davidson's present address: Department of Brain and Cognitive Sciences, Massachusetts Institute of Technology, Cambridge, MA 02140.

DOI:10.1523/JNEUROSCI.3643-04.2004

Copyright $\odot 2004$ Society for Neuroscience $\quad 0270-6474 / 04 / 2410040-07 \$ 15.00 / 0$ endogenously expressed miRNAs have been shown to regulate left-right neuronal asymmetry in Caenorhabditis elegans (Johnston and Hobert, 2003), and many more miRNAs of unknown function have been isolated as part of ribonucleoprotein complexes in rodent and human neuronal cell lines (Dostie et al., 2003). miRNAs have also been shown to be temporally regulated in neurons during mammalian brain development (Krichevsky et al., 2003; Kim et al., 2004).

Although RNAi has recently been shown to function in mammalian neurons (Gaudilliere et al., 2002; Krichevsky and Kosik, 2002; Torocsik et al., 2002), there are several specific problems that limit its usefulness in such cells. One is that the delivery procedure itself, which often uses lipid transfection reagents or electroporation, can induce neuronal damage and death and thus alter experimental outcome or interpretation. The toxicity of reagents is of particular concern in heterogeneous primary neuronal cultures, because induction of damage or death by transfection may skew the composition of the cultures toward neuronal types that are more resistant to death. Furthermore, the requirement that cells be given time to recover from transfection (typically $24-48 \mathrm{hr}$ ) means that little information is available about the earliest effects of the manipulations performed or indeed of the RNAi mechanism itself. Another limitation is that reported transfection efficiencies in neurons are typically very low, even when expressed as a fraction of cells surviving transfection. Because neurons are postmitotic, these low efficiencies cannot be countered by establishing stable cell lines. Thus, RNAi-based studies of neurons must rely on single-cell experiments rather than population-based studies, biochemical experiments, and measures of mRNA levels. Low transfection rates also limit the usefulness of RNAi for researchers who study interactions be- 
tween neurons in culture, including survival and death signals, electrical signaling in neuronal networks, cell-surface protein expression, and secreted protein expression.

Here we show that conjugates of siRNA and the vector peptide Penetratin1 (Pen1) (V-siRNA) can be used to rapidly deliver active siRNAs to primary cultures of neurons with very high efficiency and very low toxicity. The siRNA is linked to Pen 1 by a disulfide bond, which is cleaved in the reducing environment of the cytoplasm, freeing the siRNA. siRNAs delivered in this way rapidly and effectively reduce expression of specific proteins in neuronal cultures. We show that, in contrast to results seen in cell-free systems, protein knock-down mediated by perfectly complementary siRNA in neurons does not require, and can precede, target mRNA degradation.

\section{Materials and Methods}

Hippocampal neuron cultures. Hippocampi were dissected from embryonic day 18 rat fetuses, dissociated by trituration in serum-free medium, plated on polylysine $(0.1 \mathrm{mg} / \mathrm{ml})$-coated tissue culture wells or Lab-Tek (Naperville, IL) cover glass chamber slide wells, and maintained in a serum-free environment. Medium consisted of a 1:1 mixture of Eagle's MEM and Ham's F12 (Invitrogen, Carlsbad, CA) supplemented with glucose $(6 \mathrm{mg} / \mathrm{ml})$, putrescine $(60 \mu \mathrm{M})$, progesterone $(20 \mathrm{nM})$, transferrin $(100 \mu \mathrm{g} / \mathrm{ml})$, selenium $(30 \mathrm{nM})$, penicillin $(0.5 \mathrm{U} / \mathrm{ml})$, and streptomycin $(0.5 \mu \mathrm{g} / \mathrm{ml})$ (Sigma, St. Louis, MO). In all experiments, neurons were cultured for $5 \mathrm{~d}$ before treatment. Cultures contain $<2 \%$ glial cells, confirmed by staining for glial markers. For cell survival studies, indicated agents were added as described previously [3 $\mu \mathrm{M}$ 4-hydroxynonenal (HNE); Cayman Chemical, Ann Arbor, MI (Rabacchi et al., 2004); vector-linked antisense oligonucleotide targeting superoxide dismutasel (SOD1) (V-ASOD1) (Troy and Shelanski, 1994; Troy et al., 1996a)].

Sympathetic neuron cultures. Primary cultures of dissociated sympathetic neurons were prepared from the superior cervical ganglia (SCG) of postnatal day 1 mice and rats (Troy et al., 2001). Briefly, SCGs were removed from the neonates, and capsules were removed by trypsinization. Cells were resuspended in RPMI 1640 with $10 \%$ horse serum and mouse NGF (50 ng/ml) and plated on Lab-Tek cover glass chamber slides coated with Matrigel (BD Biosciences, Bedford, MA). One day after plating, uridine and 5-fluorodeoxyuridine (10 $\mu \mathrm{M}$ each) were added to the cultures and left for $4 \mathrm{~d}$ to eliminate non-neuronal cells.

siRNA design. siRNAs were designed according to published design guidelines with dTdT 3' overhangs (Elbashir et al., 2001). Sequences for the sense strand of the central 19-nt double-stranded region were: SOD1i-1, UCCUCACUCUAAGAAACAU; SOD1i-2, CCAGUGGUGGUGUCAGGAC; caspase (Casp)3i, CCUCAGAGAGACAUUCAUG; Casp8i, GCACAGAGAGAAGAAUGAG; Casp9i, GGCACCCUGGCUUCACUCU; M2i, UCUUGUCAAAUUGGACAAA. All siRNAs target the open reading frame, as shown in Figure 1.

siRNA coupling. siRNA duplexes with a $5^{\prime}$ thiol on the sense strand were synthesized and HPLC purified (Dharmacon, Lafayette, CO and Xeragon, Germantown, MD). For uptake studies, siRNAs were synthesized with a $5^{\prime}$ thiol on the sense strand and 5' FITC on the antisense strand. Annealed siRNA duplexes were resuspended in buffer provided by the manufacturer and treated with an equimolar mixture of Tris (2carboxyethyl) phosphine at $20^{\circ} \mathrm{C}$ for $15 \mathrm{~min}$. An equimolar ratio of Penetratin1 (Q-Biogene, Carlsbad, CA) was added, and the mixture heated to $65^{\circ} \mathrm{C}$ for $15 \mathrm{~min}$ and then incubated at $37^{\circ} \mathrm{C}$ for $1 \mathrm{hr}$. The yields of the reactions were estimated at $90 \%$ by SDS-PAGE using SyProRuby (Molecular Probes, Eugene, OR) (data not shown).

Transfection of hippocampal neurons. Lipofectamine 2000 (Invitrogen) was mixed with the siRNA construct according to the manufacturer's instructions and added to hippocampal neurons that had been in culture for $5 \mathrm{~d}$. Each well received $2 \mu \mathrm{l}$ of lipofectamine and 60 picomoles of siRNA in a total volume of $400 \mu \mathrm{l}$.

Immunocytochemistry. Cells were grown and treated as described above on eight-well Lab-Tek cover glass chamber slides and fixed with $4 \%$ paraformaldehyde. After a 30 min block with $3 \%$ normal goat serum,
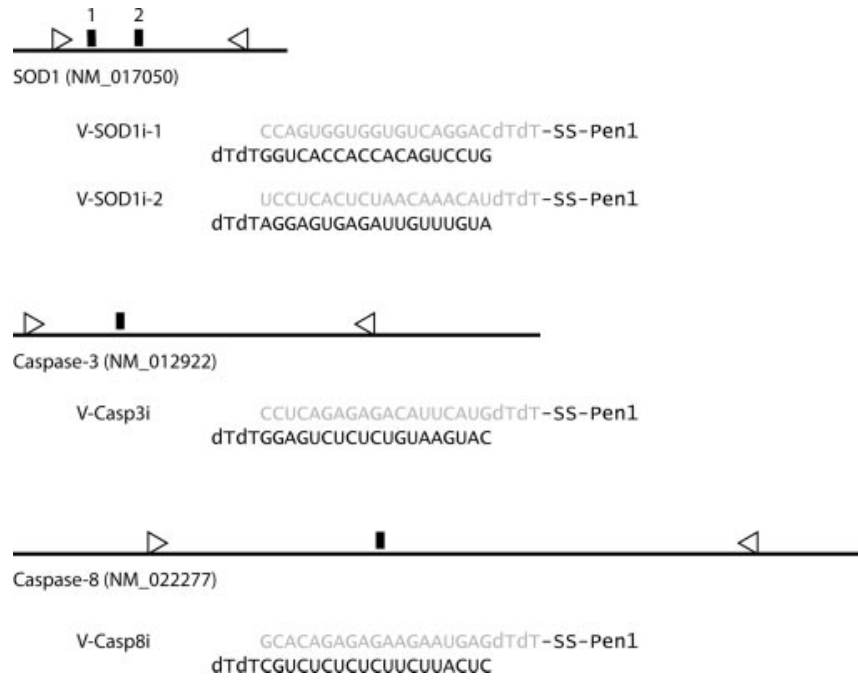

Figure 1. Scheme showing sequences and mRNA target sites of V-siRNAs. All V-siRNAs have perfect homology (black rectangles) to sites within the open reading frame (region between open triangles) of targeted mRNA. National Center for Biotechnology Information accession numbers are provided for rat mRNA. Both sense (gray; $5^{\prime}$ to $3^{\prime}$ ) and antisense (black; $3^{\prime}$ to $5^{\prime}$ ) sequences of $\mathrm{V}$-siRNAs used are shown.

slides were incubated with primary antibody for $1 \mathrm{hr}$, then washed with PBS and incubated with the appropriate secondary antibody (Alexafluor goat-anti-mouse and goat-anti-rabbit 488 and 568; Molecular Probes) for $30 \mathrm{~min}$, followed by three PBS washes. Samples were visualized with a spinning disc confocal imaging system (Perkin-Elmer, Fremont, CA).

Western blot analysis. Hippocampal cultures were harvested in sample buffer. Equal amounts of protein were separated by $4-20 \%$ PAGE, transferred to nitrocellulose membrane, and immunostained as described previously (Troy et al., 2002). Anti-SOD1 (Sigma) was used at 1:300, anti-caspase-3 (Upstate, Charlottesville, VA) at 1:1000, anti-actin at 1:1000 (Sigma), and anti-cytoplasmic inhibitor of apoptosis protein 2 (cIAP2) (MBL International, Woburn, MA) at 1:1000. Visualization was with ECL (Pierce, Rockford, IL).

SOD1 enzyme activity. Hippocampal cultures were extracted with $0.5 \%$ Nonidet NP-40, and protein was measured with the Bio-Rad (Hercules, CA) reagent. SOD levels were measured as described previously (Troy and Shelanski, 1994), with a modification of the xanthinexanthine oxidase system, measuring the reduction of nitrobluetetrazolium at $560 \mathrm{~nm}$ in the presence and absence of potassium cyanide ( $\mathrm{KCN})$. SOD1 activity was determined from an SOD standard curve and is reported as the KCN-sensitive activity.

Cell survival quantification. After removal of the medium, cultured cells were lysed in $200 \mu \mathrm{l}$ of a solution that lyses the cell membrane but leaves the nuclei intact, and intact nuclei were counted using a hemacytometer (Troy et al., 2002). Cell counts were performed in quadruplicate wells and reported as mean \pm SEM.

Quantitative PCR. For SOD1 and caspase-3, three different sets of primers were used to measure mRNA. One set of primers was designed to amplify a 300-400 base region of the targeted mRNA spanning the siRNA targeting site, one set was 5' of the targeting site, and the other was $3^{\prime}$ of the site. RNA was isolated from cultured neurons using Trizol reagent. cDNA was prepared by reverse transcriptase using SuperScript II and oligo-dT primer (Invitrogen). cDNA were added to a reaction mix (PCR ready-to-go beads; Amersham Pharmaceuticals, Buckinghamshire, UK with SYBR Green; Molecular Probes) together with appropriate primers at $0.5 \mu \mathrm{M}$ each. Levels of transcripts were analyzed using the Cepheid SmartCycler (Fisher, Pittsburgh, PA) following the manufacturer's specifications. RNA controls were used to ensure that amplification of products did not come from genomic DNA. PCR products for SOD1 SmartCycling were sequenced by the DNA sequencing facility of Columbia University (New York, NY) and were confirmed to be identical to the expected sequence for SOD1. Melt curve analysis was used to determine 
the temperature for fluorescence detection. For each mRNA, quantification was made from the linear portion of the amplification curve. $\alpha$-Tubulin mRNA levels were used to normalize input cDNA.

\section{Results}

V-siRNA is rapidly and efficiently taken up by neurons

To determine whether the V-siRNA was efficiently delivered to the cytoplasm of neurons as an intact duplex, we synthesized an siRNA with a fluorescein group at the $5^{\prime}$ end of the antisense strand and a thiol group on the $5^{\prime}$ end of the sense strand (base composition of siRNAs are detailed in Materials and Methods). This siRNA was linked to Pen1 by a disulfide bond (V-siRNAFITC) (Fig. 2a); the disulfide bond is reduced after delivery to the cytoplasm. We monitored uptake in live primary cultures of hippocampal neurons by confocal microscopy and observed significant uptake of V-siRNA-FITC within $15 \mathrm{~min}$. At $2 \mathrm{hr}$ of treatment, the medium was replaced with fresh medium lacking V-siRNA-FITC. Essentially all neurons in the primary rat hippocampal cultures treated with V-siRNA-FITC showed extensive punctate cytoplasmic fluorescence ( $99 \%$ of cells showed uptake) (Fig. 2b, top). Unlinked FITC-labeled siRNA (siRNA-FITC) was not taken up by the neurons (Fig. $2 b$, bottom). In all subsequent experiments, Pen 1 was linked to the siRNA by a thiol group on the $5^{\prime}$ end of the sense strand, whereas the antisense strand of the siRNA was unmodified.

Cell damage and death can lead to alterations in the composition and functional properties of complex heterogeneous cultures. We therefore compared the cell toxicity of vector-linked siRNA with that of the transfection reagent Lipofectamine 2000. The latter has been reported to successfully deliver siRNA to primary neuronal cultures (Krichevsky and Kosik, 2002; Fink et al., 2003; Omi et al., 2004). The siRNA was targeted against caspase- 3 and anticipated to have no harmful cellular effects of its own, based on our previous studies using antisense oligonucleotides (Troy et al., 2001). Hippocampal cultures were evenly plated and cultured for $5 \mathrm{~d}$ before treatment. Live cells were imaged by phase microscopy at $0,6,24$, and $48 \mathrm{hr}$ after treatment and lysed and counted at 0,24 , and $48 \mathrm{hr}$ (Fig. $2 c$ ). Neurons in the transfected cultures showed evidence of injury: by $6 \mathrm{hr}$ there was substantial debris in the medium, neurites were beginning to degenerate, and some neuronal cell bodies had vacuoles. None of these effects were seen in the control or V-siRNA-treated cultures. At $24 \mathrm{hr}$ after treatment, there were fewer neurons in the transfected cultures, and the remaining neurons had fewer neurites per cell than cells in control cultures. Neurons in cultures treated with $\mathrm{V}$-siRNA were qualitatively indistinguishable from untreated control cultures at all time points. Survival at $24 \mathrm{hr}$, relative to control at $0 \mathrm{hr}$, was $99 \%$ for control, $92 \%$ for $\mathrm{V}$-siRNA-treated cells, and 58\% for cells transfected with Lipofectamine 2000 (Fig. 2c). At $48 \mathrm{hr}$ after treatment, no significant additional cell death was observed, consistent with an acute insult caused by the transfection. By 48 hr, however, transfected cells had not regenerated their extensive network of processes.

\section{V-siRNA knocks down levels of targeted endogenous proteins within hours}

Immunocytochemistry revealed that treatment with $\mathrm{V}$-siRNAs at a concentration of $80 \mathrm{nM}$ was effective in knocking down levels of the targeted endogenous proteins at 5-6 hr after treatment in neuronal cultures derived from sympathetic ganglia and hippocampus (Fig. 3). Cultures were treated with the indicated $\mathrm{V}$-siRNA for $6 \mathrm{hr}$, fixed, and immunostained for the target protein and a nontargeted protein. In all cases, the targeted protein

a

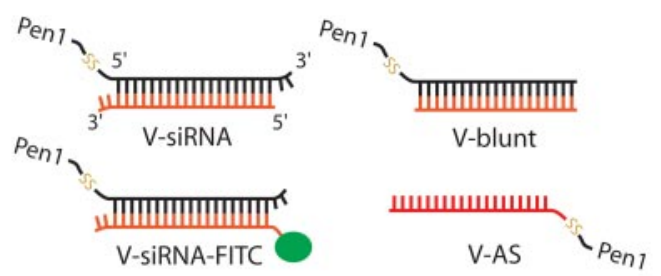

b

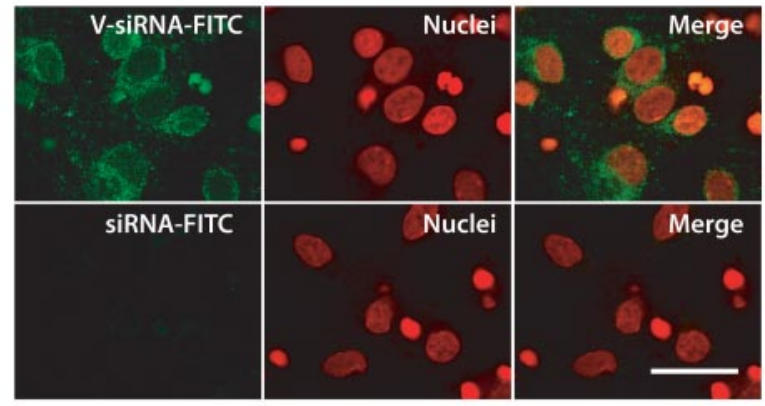

C

Control

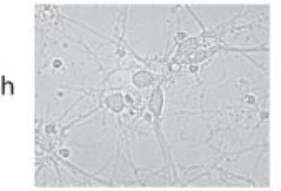

$6 \mathrm{~h}$
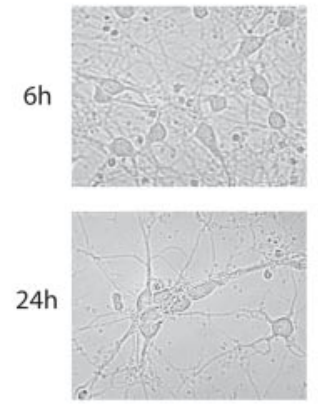

Survival:

$24 \mathrm{~h}$

$48 \mathrm{~h}$

$(\% \pm S D)$

\section{Figure} Scheme for linking vector (Penetratin1) to constructs: V-siRNA, V-siRNA-FITC, V-blunt, and vector-linked antisense oligonucleotides (V-AS). $b$, Neuronal uptake of V-siRNA. Primary cultures of hippocampal neurons were treated with fluorescein-labeled siRNA (bottom) or vectorlinked siRNA-FITC (top), shown in green. DRAQ5, shown in red, was added to visualize nuclei. Live cells were imaged every $15 \mathrm{~min}$; images shown were taken at $2 \mathrm{hr}$. Uptake of V-siRNA-FITC was shown by $99 \pm 3 \%$ of cells $(n=4)$. Smaller cells are cells that are not fully in the plane of focus. Scale bar, $50 \mu \mathrm{m}$. c, Phase micrographs of hippocampal cultures after siRNA delivery. Treatment of hippocampal neurons with V-siRNA causes minimal morbidity-mortality. Hippocampal cultures were untreated (Control), treated with Lipofectamine 2000 and siRNA for Casp3 (Transfected), or treated with V-Casp3i (V-siRNA). Live cultures were imaged at 0, 6, and $24 \mathrm{hr}$. Three control wells were lysed and counted at $0 \mathrm{hr}$, and remaining cultures were counted at 24 and $48 \mathrm{hr}$ ( $n=4$ wells per treatment and time point). Survival is reported at 24 and $48 \mathrm{hr}$ relative to the number of cells per well present at time 0 . Scale bar, $50 \mu \mathrm{m}$.

(green) was downregulated and the nontargeted protein (red) was not. Two siRNAs targeting $\mathrm{Cu}-\mathrm{Zn}$ SOD1 were effective. Targeting single members of the caspase family separately (we targeted caspases-3, -8, and -9) did not affect other, nontargeted, caspase family members.

We next quantified the extent of knock-down of selected proteins at $6 \mathrm{hr}$ after treatment by a range of V-siRNA concentra- 

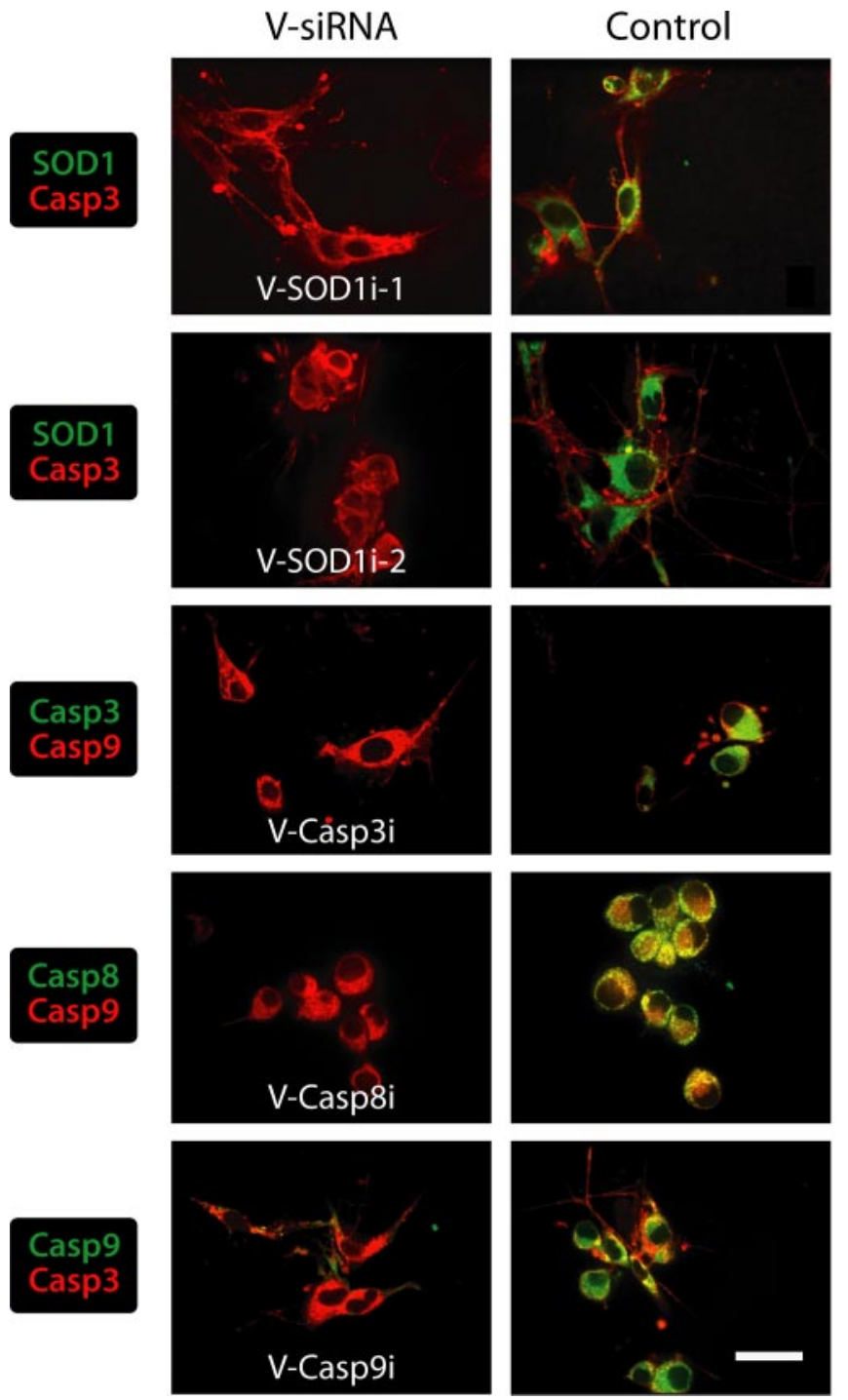

Figure 3. V-siRNAs specifically suppress endogenous protein expression. Neuronal cultures (V-SOD1i-1/2, sympathetic; other V-siRNAs, hippocampal) were treated with the indicated V-siRNA at $80 \mathrm{~nm}$ and fixed at 5-6 hr. Untreated control cultures are shown on the right. Cultures were immunostained for the target protein (green) and a nontargeted protein (red), as indicated. Scale bar, $50 \mu \mathrm{m}$.

tions. SOD1 was measured by an enzyme activity assay and by Western blotting (Fig. 4a,b). By both measures, the knock-down in response to treatment with $\mathrm{V}$-siRNA targeting SOD1 (VSOD1i-1) was dose dependent and nearly complete at $80 \mathrm{~nm}$ (protein $10 \%$ of control; enzyme activity 0 ). Measurement of levels of caspase-3 protein by Western blotting showed a similar dependence on concentration of V-siRNA targeting caspase-3 (V-Casp3i), with $80 \mathrm{~nm}$ knocking protein down to $27 \%$ of control and $320 \mathrm{~nm}$ to $3 \%$ of control. The time course of knock-down for both of these proteins is in agreement with our previous work in neuronal cells using antisense oligonucleotides (Troy and Shelanski, 1994; Troy et al., 1996a, 2001). To confirm that the protein knock-down we observed was functionally relevant, we replicated previous studies in which antisense oligonucleotides were delivered to neurons either to protect from or to induce apoptotic death. Knock-down of SOD1 induces an interleukin$1 \beta$-dependent, nitric oxide-mediated neuronal apoptosis (Troy et al., 1996a,b). We show that application of V-SOD1i-1 or V-SOD1i-2 to hippocampal neurons leads to a dose-dependent death (Fig. $4 d$, red and orange lines). This effect is greater at all concentrations for the V-siRNAs than for V-ASOD1 (Fig. $4 d$, green line). An unrelated V-siRNA (V-M2i) (Fig. 4d, blue line) did not induce death. We also used V-siRNA to protect neurons from apoptosis. Caspase-3 is a critical effector of HNE-induced death; knock-down of caspase-3 protects neurons from HNE (Rabacchi et al., 2004). V-Casp3i was able to rescue hippocampal neurons from HNE-induced apoptosis in a dose-dependent manner with full protection by $320 \mathrm{~nm}$ (Fig. $4 e$, red line).

\section{Protein knock-down does not require, and can precede,} mRNA degradation

Given that RNAi in mammals is thought to result from the sequence-specific cleavage of homologous mRNA, we next examined the effect of $\mathrm{V}$-siRNA on the levels of targeted mRNA. Total RNA was harvested from cultures, and Northern blot analysis was performed. Surprisingly, at time points corresponding to nearly complete downregulation of targeted proteins (6 hr), corresponding mRNA levels were unchanged (data not shown). Realtime PCR was performed to further quantify mRNA levels. For each target mRNA, we used three different sets of primers, including one pair that spanned the regions targeted by our siRNAs. All primer pairs yielded similar results. SOD1 protein knock-down at $6 \mathrm{hr}$ had been confirmed by immunocytochemistry, Western blotting, and enzyme activity assays, but mRNA levels at this time point were unchanged, even by treatment at high concentrations of V-SOD1i-1 (Fig. 4f, unfilled red bars). PCR products were sequenced and were identical across conditions and to the rat SOD1 sequence. By $24 \mathrm{hr}$ after treatment, however, SOD1 mRNA levels had declined by $>80 \%$ (Fig. $4 f$, filled red bars). In contrast to the effect on protein observed at 6 $\mathrm{hr}$, there was no significant difference in mRNA levels between concentrations of 8,80 , and $320 \mathrm{nM}$, suggesting that the mechanism responsible for mRNA degradation was saturated at even the lowest concentration. Another siRNA targeting SOD1, V-SOD1i-2, showed knock-down of protein expression (Fig. 3) and effectively induced hippocampal death (Fig. $4 d$ ) but did not alter mRNA levels of SOD 1 at 6 or $24 \mathrm{hr}$ (Fig. $4 f$, yellow bars). An unrelated V-siRNA also did not alter SOD1 mRNA levels (VCasp3i) (Fig. $4 f$, blue bars). Levels of mRNA encoding proteins unrelated to SOD1 (cIAP2, caspase-2, and Fas ligand) were not altered by any of these treatments (data not shown). Similarly, V-Casp3i did not downregulate caspase-3 mRNA at 6 or $24 \mathrm{hr}$ (Fig. $4 g$, red bars), although protein was knocked down by $6 \mathrm{hr}$ (Fig. 4c), and functional effects were apparent at $24 \mathrm{hr}$ (Fig. 4e). V-SOD1i-1 did not alter caspase-3 mRNA levels (Fig. $4 g$, blue bars), showing that its effects were specific for the SOD1 message. Thus, although vector-delivered siRNAs showed specific effects on protein levels and function within $24 \mathrm{hr}$, they did not show consistent effects on corresponding mRNA levels within this time frame.

\section{Both early protein and late mRNA effects require $3^{\prime}$ \\ 2-nt overhang}

We next considered whether the early protein knock-down we observed with V-siRNA could be attributable to an antisense rather than an RNAi effect. Because siRNAs are linked to Pen 1 by their sense strand, we know that all antisense strands delivered to the cytoplasm must be delivered as part of intact duplexes. Furthermore, our functional results show that, consistent with the first observations of RNAi (Fire et al., 1998), V-siRNA is effective at much lower concentrations than vector-linked antisense oligonucleotides (Fig. 4d). 
To test whether V-siRNA might work by an antisense mechanism, we took advantage of the fact that incorporation into the RNAi-inducing silencing complex (RISC) requires a 2-nucleotide 3 ' overhang on both ends of the siRNA (Elbashir et al., 2001). No such requirement exists for antisense oligonucleotides, which are delivered to cells as single-stranded DNA or RNA and are thought to inhibit translation by steric hindrance or $\mathrm{RNaseH}$ activation (Vickers et al., 2003). We used vectorlinked RNA duplexes that had the same sequences as V-SOD1i-1 and V-Casp3i over the central double-stranded portion of the siRNA but lacked the 2-nt 3' overhang (V-blunt) (Fig. 2a). Treatment with $\mathrm{V}$-blunt in the same concentrations as $\mathrm{V}$-siRNA had no effect on either protein (Fig. $4 b, c$ ) or message (Fig. 4f,g, gray bars) and did not have functional effects (Fig. $4 d$,e, gray lines), suggesting that the actions of $\mathrm{V}$-siRNAs were not a result of an antisense effect. The rapid protein knockdown in the absence of consistent mRNA degradation supports an miRNA-like translational suppression mechanism.

\section{Discussion}

Neurons have historically proven refractory to easy genetic manipulation. They are more resistant to transfection than most other cell types and, because they are postmitotic, stable mutant cell lines cannot be established to counter these low efficiencies. Here we show that treatment with vector peptide-linked siRNAs leads to highly efficient induction of RNAi in mammalian neurons cultured from the central and peripheral nervous systems. We also show that a widely used lipid transfection reagent induces substantial morbidity and mortality in the neuronal cultures, whereas the Pen 1 delivery system has minimal toxic effects. Pen 1, a 16 amino acid peptide corresponding to the third helix of the Antennapedia homeodomain protein, enters cells in a non-receptor-mediated manner (Derossi et al., 1994; Theodore et al., 1995; Joliot and Prochiantz, 2004). We have previously used Pen1 to deliver antisense oligonucleotides (Troy et al., 1996a, 2001, 2002) and peptide cargoes (Troy et al., 1996b) to primary neurons in culture to study the molecular mechanisms of neuronal death. Highly efficient facilitated delivery also allows the use of low concentrations of siRNA, which likely reduces potential nonspecific side effects while being cost effective.

The combination of new, mechanism-based design rules for siRNAs (Khvorova et al., 2003; Schwarz et al., 2003) and our simple, nontoxic method of inducing RNAi in neurons opens up the possibility, already realized in areas outside of neurobiology, of exploiting large public libraries of siRNAs for use in genetic screens. Preliminary experiments have also shown this technique to be amenable to performing simultaneous knock-downs of multiple proteins (data not shown). The high efficiencies and
SOD1
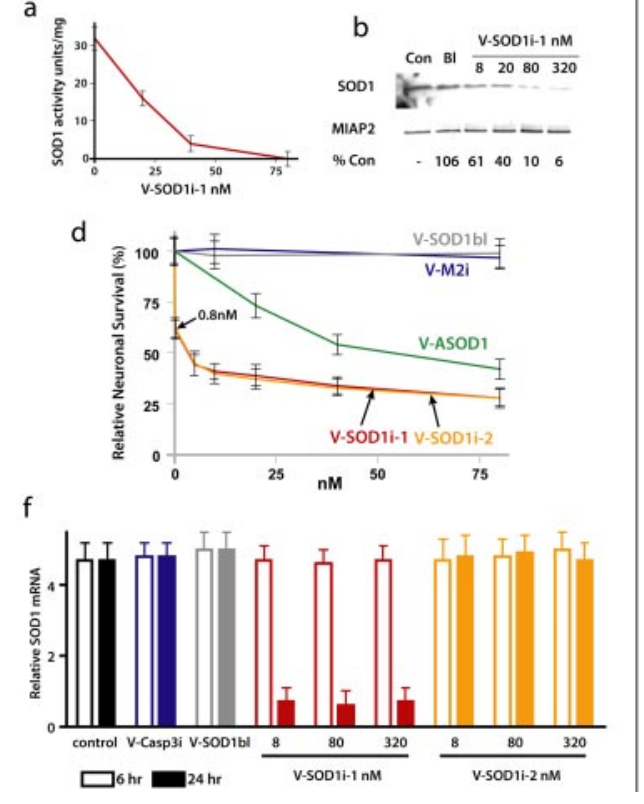

Caspase-3

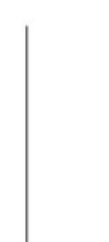

e
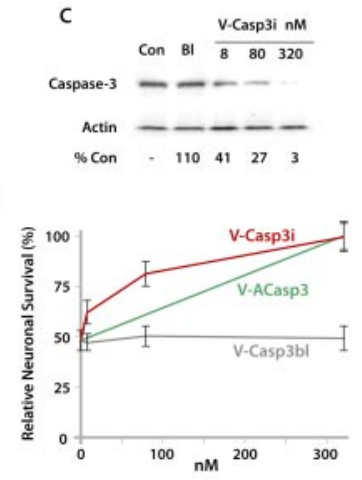

g

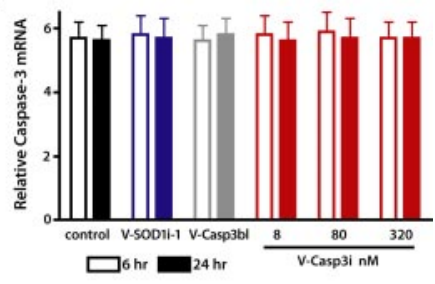

Figure 4. Translational suppression by V-siRNA precedes mRNA downregulation. Throughout this figure: red and orange,

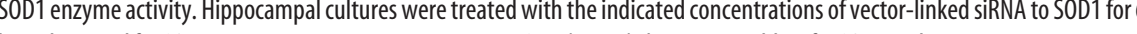
hr and assayed for SOD1 enzyme activity, given as mean \pm SEM $(n=3) . b, c$, Western blots for SOD1 and caspase-3. Hippocampal 作 protein 2 (MIAP2) or actin to control for loading. S0D1 and caspase-3 levels are normalized to MIAP2 or actin and expressed as percentage of control, as determined by densitometry. $d$, V-SOD1i induces hippocampal neuronal death and does so more effectively than antisense oligonucleotides. Hippocampal cultures were treated with V-SOD1i-1/2, V-ASOD1, blunt-ended V-S0D1i-1 (V-SOD1bl), or unrelated vector-linked siRNA (V-M2i). Survival was assessed after $1 \mathrm{~d}$ by counting nuclei in cell lysates.

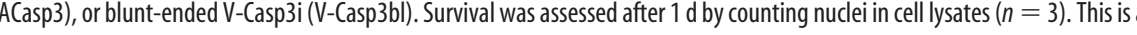
(D) cultures were treated with the indicated constructs, and RNA was harvested after 6 (open bars) and $24 \mathrm{hr}$ independent runs. Results were normalized to $\alpha$-tubulin mRNA levels and are given as the mean $\pm \operatorname{SEM}(n=3)$. Similar results were obtained when normalizing against caspase- 2 mRNA levels.

minimal toxicity of V-siRNA treatment bring multiple benefits: they allow for the use of population-wide assays of changes in biological activity and biochemical composition, and they bring the power of RNAi to the study of systems involving interactions between neurons. This method also facilitates studies of cell signaling, particularly of pathways mediating survival and death, without artifactual interference from the delivery method, as may occur with transfection. During the preparation of this manuscript, a study was published showing that Pen1-siRNA conjugates can also be used in non-neuronal cell lines to suppress expression of exogenous mRNA (Muratovska and Eccles, 2004). We have confirmed that Pen 1 can be used to deliver siRNA to non-neuronal cells (data not shown), but find that the cellular responses may differ in neurons and non-neuronal cells (see below).

The rapid uptake of V-siRNA and the ability to study treated cultures without having to allow them to recover from transfection have permitted us to study effects of siRNA on targeted endogenous protein and message levels at earlier time points than was previously possible in cultured mammalian cells. The effects 
we see on the protein levels are in agreement with our studies using antisense oligonucleotides for these target proteins (Troy and Shelanski, 1994; Troy et al., 2001) and with the effects on the enzyme activity of these target proteins (Fig. 4) (Rabacchi et al., 2004), suggesting that the half-lives of the target proteins are $<5$ $\mathrm{hr}$ in cultured hippocampal neurons. In a Drosophila cell-free model system, cleavage of mRNA has been shown to occur as soon as 5 min after introduction of siRNA (Schwarz et al., 2003). However, all published data on RNAi in neurons has been obtained at least $24 \mathrm{hr}$ after treatment.

A consensus model of the siRNA-miRNA pathway that is often proposed is one in which imperfect matching to the target mRNA (as seen in many endogenous miRNAs) leads to translational repression, whereas perfect complementarity (as in most synthetic siRNAs) leads to message degradation (Lai, 2003; Scherer and Rossi, 2003; Bartel, 2004). This model is supported by the ability of imperfectly matched exogenous siRNAs to function as miRNAs (Doench et al., 2003; Saxena et al., 2003) and of miRNA to induce mRNA cleavage of perfectly complementary targets, as demonstrated recently in human cell lines (Llave et al., 2002; Zeng et al., 2003). Here we show that, in neurons, perfectly matching siRNA can specifically downregulate targeted proteins with no effect on targeted message. We also used the V-SOD1i-1 construct in Rat1 fibroblasts and find downregulation of mRNA and protein by $6 \mathrm{hr}$ of treatment (data not shown), indicating that there may be differences in the actions of siRNA in neuronal and non-neuronal cells. All siRNAs used target the open reading frame of the targeted messages (Fig. 1). This suggests novel models of the effects of siRNA in neurons. One possible model is that a RISC capable of miRNA-like translational repression may be immediately assembled after introduction of siRNA, but that this complex may not be capable of catalytically cleaving mRNA until some unspecified triggering event occurs or cofactor becomes available. Another possibility is that the miRNA-like translational repression may proceed by an independent pathway with different (but potentially overlapping) enzymatic and complementarity requirements.

The siRNAs used in this experiment were designed before recent work showed the dependence of message cleaving ability on low bond strength at the $5^{\prime}$ end of the antisense strand of the siRNA (Khvorova et al., 2003; Schwarz et al., 2003) as well as the presence of the base $U$ at position 10 (Reynolds et al., 2004). However, a post hoc analysis shows that the only siRNA we found to have an effect on message levels (V-SOD1i-1) meets these requirements, with an $\mathrm{A}: \mathrm{U}$ bond at three of the first four positions of the antisense strand (AUGU... ), including the critical first position, and a $U$ at position 10 . In contrast, siRNAs that had early effects on protein levels but no effect on mRNA by $24 \mathrm{hr}$, had higher free energy at the $5^{\prime}$ end of the antisense strand (VSOD1i-2, GUCC... ; V-Casp3i, CAUG. . ; see Materials and Methods), and did not have a $U$ at position 10. Because these free-energy requirements are thought to determine whether an siRNA will be incorporated into the RISC, and not how efficiently the assembled RISC will cleave its target mRNA (Khvorova et al., 2003; Schwarz et al., 2003), this observation favors an independent pathway for the observed early effects on protein. On the other hand, the finding that early protein knock-down requires siRNA with 2-nt 3' overhangs suggests that even siRNAs that do not eventually mediate mRNA cleavage are being recognized and incorporated into siRNA-specific silencing complexes.

We also observed that early protein knock-down shows a dose dependence markedly different from that observed for message cleavage. Whereas mRNA degradation was saturated by V-siRNA at concentrations of $8 \mathrm{nM}$, the early protein knock-down was not saturated even by concentrations of $80 \mathrm{~nm}$. The dose dependence of the early miRNA-like effect suggests that it may not be acting catalytically, unlike traditional RNAi involving cleavage of message (Doench et al., 2003). This observation is consistent with both of the models proposed above.

The ability to deliver siRNA to neurons simply, efficiently, and without toxicity provides a much-needed tool for researchers to study and understand endogenous protein function as well as the mechanisms of RNAi itself. Mounting evidence suggests that these mechanisms also likely play an important role in the normal function and development of the nervous system. An additional intriguing possibility is that small double-stranded RNAs may play a role in the pathogenesis and treatment of genetic diseases. Genetic polymorphisms that are normally suppressed by miRNAs may be expressed when a suppressor miRNA is absent, leading to expression of mutant protein and development of disease. With respect to treatment, siRNAs could be delivered therapeutically as sequence-specific suppressors of particular protein isoforms. The vector-peptide Pen 1 has been used for the delivery of cargoes in vivo (Bertrand et al., 2001), and the method described here could potentially be adapted for nontoxic in vivo delivery of siRNA, providing for additional mechanistic studies and potential gene-therapeutic applications.

\section{References}

Bartel DP (2004) MicroRNAs: genomics, biogenesis, mechanism, and function. Cell 116:281-297.

Bertrand E, Brouillet E, Caille I, Bouillot C, Cole GM, Prochiantz A, Allinquant B (2001) A short cytoplasmic domain of the amyloid precursor protein induces apoptosis in vitro and in vivo. Mol Cell Neurosci 18:503-511.

Derossi D, Joliot AH, Chassaing G, Prochiantz A (1994) The third helix of the Antennapedia homeodomain translocates through biological membranes. J Biol Chem 269:10444-10450.

Doench JG, Petersen CP, Sharp PA (2003) siRNAs can function as miRNAs. Genes Dev 17:438-442.

Dostie J, Mourelatos Z, Yang M, Sharma A, Dreyfuss G (2003) Numerous microRNPs in neuronal cells containing novel microRNAs. RNA 9:180-186.

Elbashir SM, Martinez J, Patkaniowska A, Lendeckel W, Tuschl T (2001) Functional anatomy of siRNAs for mediating efficient RNAi in Drosophila melanogaster embryo lysate. EMBO J 20:6877-6888.

Fink CC, Bayer KU, Myers JW, Ferrell Jr JE, Schulman H, Meyer T (2003) Selective regulation of neurite extension and synapse formation by the beta but not the alpha isoform of CaMKII. Neuron 39:283-297.

Fire A, Xu S, Montgomery MK, Kostas SA, Driver SE, Mello CC (1998) Potent and specific genetic interference by double-stranded RNA in Caenorhabditis elegans. Nature 391:806-811.

Gaudilliere B, Shi Y, Bonni A (2002) RNA interference reveals a requirement for myocyte enhancer factor $2 \mathrm{~A}$ in activity-dependent neuronal survival. J Biol Chem 277:46442-46446.

Hannon GJ (2002) RNA interference. Nature 418:244-251.

Hutvagner G, Zamore PD (2002) A microRNA in a multiple-turnover RNAi enzyme complex. Science 297:2056-2060.

Johnston RJ, Hobert O (2003) A microRNA controlling left/right neuronal asymmetry in Caenorhabditis elegans. Nature 426:845-849.

Joliot A, Prochiantz A (2004) Transduction peptides: from technology to physiology. Nat Cell Biol 6:189-196.

Khvorova A, Reynolds A, Jayasena SD (2003) Functional siRNAs and miRNAs exhibit strand bias. Cell 115:209-216.

Kim J, Krichevsky A, Grad Y, Hayes GD, Kosik KS, Church GM, Ruvkun G (2004) Identification of many microRNAs that copurify with polyribosomes in mammalian neurons. Proc Natl Acad Sci USA 101:360-365.

Krichevsky AM, Kosik KS (2002) RNAi functions in cultured mammalian neurons. Proc Natl Acad Sci USA 99:11926-11929.

Krichevsky AM, King KS, Donahue CP, Khrapko K, Kosik KS (2003) A microRNA array reveals extensive regulation of microRNAs during brain development. RNA 9:1274-1281. 
Lai EC (2003) microRNAs: runts of the genome assert themselves. Curr Biol 13:R925-936.

Llave C, Xie Z, Kasschau KD, Carrington JC (2002) Cleavage of Scarecrowlike mRNA targets directed by a class of Arabidopsis miRNA. Science 297:2053-2056.

Muratovska A, Eccles MR (2004) Conjugate for efficient delivery of short interfering RNA (siRNA) into mammalian cells. FEBS Lett 558:63-68.

Omi K, Tokunaga K, Hohjoh H (2004) Long-lasting RNAi activity in mammalian neurons. FEBS Lett 558:89-95.

Rabacchi SA, Friedman WJ, Shelanski ML, Troy CM (2004) Divergence of the apoptotic pathways induced by 4-hydroxynonenal and amyloid betaprotein. Neurobiol Aging 25:1057-1066.

Reynolds A, Leake D, Boese Q, Scaringe S, Marshall WS, Khvorova A (2004) Rational siRNA design for RNA interference. Nat Biotechnol 22:326-330.

Saxena S, Jonsson ZO, Dutta A (2003) Small RNAs with imperfect match to endogenous mRNA repress translation. Implications for off-target activity of small inhibitory RNA in mammalian cells. J Biol Chem 278:44312-44319.

Scherer LJ, Rossi JJ (2003) Approaches for the sequence-specific knockdown of mRNA. Nat Biotechnol 21:1457-1465.

Schwarz DS, Hutvagner G, Du T, Xu Z, Aronin N, Zamore PD (2003) Asymmetry in the assembly of the RNAi enzyme complex. Cell 115:199-208.

Theodore L, Derossi D, Chassaing G, Llirbat B, Kubes M, Jordan P, Chneiweiss H, Godement P, Prochiantz A (1995) Intraneuronal delivery of protein kinase $\mathrm{C}$ pseudosubstrate leads to growth cone collapse. J Neurosci 15:7158-7167.

Torocsik B, Angelastro JM, Greene LA (2002) The basic region and leucine zipper transcription factor MafK is a new nerve growth factor-responsive immediate early gene that regulates neurite outgrowth. J Neurosci 22:8971-8980

Troy CM, Shelanski ML (1994) Down-regulation of copper/zinc superoxide dismutase causes apoptotic death in PC12 neuronal cells. Proc Natl Acad Sci USA 91:6384-6387.

Troy CM, Derossi D, Prochiantz A, Greene LA, Shelanski ML (1996a) Downregulation of $\mathrm{Cu} / \mathrm{Zn}$ superoxide dismutase leads to cell death via the nitric oxide-peroxynitrite pathway. J Neurosci 16:253-261.

Troy CM, Stefanis L, Prochiantz A, Greene LA, Shelanski ML (1996b) The contrasting roles of ICE family proteases and interleukin-1beta in apoptosis induced by trophic factor withdrawal and by copper/zinc superoxide dismutase down-regulation. Proc Natl Acad Sci USA 93:5635-5640.

Troy CM, Rabacchi SA, Hohl JB, Angelastro JM, Greene LA, Shelanski ML (2001) Death in the balance: alternative participation of the caspase-2 and -9 pathways in neuronal death induced by nerve growth factor deprivation. J Neurosci 21:5007-5016.

Troy CM, Friedman JE, Friedman WJ (2002) Mechanisms of p75-mediated death of hippocampal neurons: role of caspases. J Biol Chem 277:34295-34302.

Vickers TA, Koo S, Bennett CF, Crooke ST, Dean NM, Baker BF (2003) Efficient reduction of target RNAs by small interfering RNA and RNase $\mathrm{H}$-dependent antisense agents. A comparative analysis. J Biol Chem 278:7108-7118.

Zeng Y, Yi R, Cullen BR (2003) MicroRNAs and small interfering RNAs can inhibit mRNA expression by similar mechanisms. Proc Natl Acad Sci USA 100:9779-9784. 